Pacific Journal of Mathematics

FINITELY GENERATED PROJECTIVE MODULES AND TI 


\title{
FINITELY GENERATED PROJECTIVE MODULES AND TTF CLASSES
}

\author{
Robert W. Miller
}

\begin{abstract}
Let $P$ be a finitely generated projective right $A$-module with trace ideal $T$ and $A$-endomorphism ring $B$. Associated with $P$ are the TTF classes, $\mathscr{T}_{F}=\left\{{ }_{A} X \mid P \otimes X=0\right\}$ and $\mathscr{T}_{H}=$ $\left\{X_{A} \mid \operatorname{Hom}(P, X)=0\right\}$. An investigation of these TTF classes yields characterizations of various conditions on $P$ and $T$; e.g., (1) ${ }_{B} P$ is projective (flat) and (2) ${ }_{A} T$ is projective (flat). The concept of weak stability for a hereditary torsion class is introduced and characterizations are given.
\end{abstract}

A small portion of this paper was taken from the author's doctoral dissertation, under the direction of Professor F. L. Sandomierski, at the University of Wisconsin. The author is indebted to Professor Sandomierski for his guidance and encouragement.

1. Preliminaries. In this paper all rings will be associative with unit and all modules will be unitary. $E(M)$ will denote the injective hull of a module $M$. Given a ring $A$ the category of all left (right) $A$-modules will be denoted by ${ }_{A} \mathcal{M}\left(\mathcal{M}_{A}\right)$.

A familiarity with torsion theories and their terminology is assumed. For further information the reader is referred to [5] or [14]. Given a hereditary torsion class $\mathscr{T}$, its associated idempotent topologizing filter will be denoted by $f(\mathscr{T})$. We let $t(X)$ denote the torsion submodule of a module $X$.

Jans [7] has called a torsion class $\mathscr{T}$ which is also a torsionfree class for some torsion class $\mathscr{C}$, a torsion-torsionfree (TTF) class. In this case we have a TTF-theory $(\mathscr{C}, \mathscr{T}, \mathscr{F})$. In [7] it is shown there is a one-to-one correspondence between the TTF classes of ${ }_{A} \mathcal{M}$ and the idempotent ideals of $A$ given by $\mathscr{T} \rightarrow T=c(A)$, the $\mathscr{C}$-torsion submodule of $A$. The inverse correspondence is given by $T \rightarrow \mathscr{T}=\left\{{ }_{A} X \mid T X=0\right\}$. One easily checks that $\mathscr{C}=\left\{{ }_{A} X \mid A / T \otimes X=0\right\}, \quad \mathscr{F}=$ $\left\{{ }_{A} X \mid \operatorname{Hom}(A / T, X)=0\right\}$, and $T$ is the smallest element in $f(\mathscr{T})$ (i.e., $T \in f(\mathscr{T})$ and $T \subseteq I$ for all $I \in f(\mathscr{T})$ ).

For an $A$-module $U$, we say that an $A$-module $X$ is of $U$-dominant codimension $\geqq n$ (written $U$.dom.codim. $X \geqq n$ ) if there is an exact sequence

$$
X_{n} \rightarrow \cdots \rightarrow X_{1} \rightarrow X \rightarrow 0
$$

where each $X_{i}$ is a direct sum of copies of $U$. This definition is dual to 
the definition of $V$-dominant dimension given in [12]. We shall let $\mathscr{C}_{n}\left(U_{A}\right)\left(\mathscr{D}_{n}\left(V_{A}\right)\right)$ represent the full subcategory of $\mathcal{M}_{A}$ consisting of all $A$-modules of $U$-dominant codimension $\geqq n$ ( $V$-dominant dimension $\geqq n)$. Clearly for all $n \geqq 1, \mathscr{D}_{n+1}\left(V_{A}\right) \subseteq \mathscr{D}_{n}\left(V_{A}\right)$ and $\mathscr{C}_{n+1}\left(U_{A}\right) \subseteq \mathscr{C}_{n}\left(U_{A}\right)$.

It is well known that $(\mathscr{T}, \mathscr{F}) \subseteq{ }_{A} \mathcal{M}$ is a hereditary torsion theory if and only if $(\mathscr{T}, \mathscr{F})$ is cogenerated by an injective module ${ }_{A} V$; that is, $\mathscr{T}=\left\{{ }_{A} X \mid \operatorname{Hom}(X, V)=0\right\}$ and $\mathscr{F}=\mathscr{D}_{1}\left({ }_{A} V\right)$. By [12, Lemma 5.3] $Y \in \mathscr{D}_{2}\left({ }_{A} V\right)$ if and only if $Y$ is torsionfree and $\mathscr{T}$-injective.

LEMMA 1.1. Let $\mathscr{T}$ be the hereditary torsion class cogenerated by the injective module ${ }_{A} V$. The following are equivalent.

(1) ${ }_{A} Y \in \mathscr{D}_{n}\left({ }_{A} V\right)$

(2) $\operatorname{Ext}^{k}(A / I, Y)=0$ for all $0 \leqq k<n$ and for all $I \in f(\mathscr{T})$.

Remark. If $\mathscr{T}$ is a TTF class and $T$ is the smallest element in $f(\mathscr{T})$ then (2) may be replaced by $\operatorname{Ext}^{k}(A / T, Y)=0$ for all $0 \leqq k<n$.

Proof. By [14, Proposition 2.8] $Y \in \mathscr{D}_{1}\left({ }_{A} V\right)=\mathscr{F}$ if and only if $\operatorname{Hom}(A / I, Y)=0$ for all $I \in f(\mathscr{T})$. For $n>1, Y \in \mathscr{D}_{n}\left({ }_{A} V\right)$ if and only if there is an exact sequence

$$
0 \rightarrow Y \rightarrow M \rightarrow N \rightarrow 0
$$

where $M$ is a direct product of copies of $V$ and $N \in \mathscr{D}_{n-1}\left({ }_{A} V\right)$. The result follows by an easy induction.

Let $P_{A}$ be projective with trace ideal $T$ (see [1]) and $B=\operatorname{End}\left(P_{A}\right)$. The functors $F=P \otimes_{A}(\quad):{ }_{A} \mathcal{M} \rightarrow{ }_{B} \mathcal{M}$ and $H=\operatorname{Hom}\left(P_{A}, \quad\right): \mathcal{M}_{A} \rightarrow \mathcal{M}_{B}$ yield TTF classes $\mathscr{T}_{F}=\left\{{ }_{A} X \mid F\left({ }_{A} X\right)=0\right\}$ and $\mathscr{T}_{H}=\left\{X_{A} \mid H\left(X_{A}\right)=0\right\}$. It is easy to check that $T$ is the smallest element in both filters $f\left(\mathscr{T}_{F}\right)$ and $f\left(\mathscr{T}_{H}\right)$, and that $\mathscr{C}_{H}=\mathscr{C}_{1}\left(P_{A}\right)$.

Lemma 1.1*. Let $\mathscr{T}_{H}$ be the TTF class generated by the projective module $P_{A}$. The following are equivalent.

(1) $X_{A} \in \mathscr{C}_{n}\left(P_{A}\right)$.

(2) $\operatorname{Tor}_{k}(X, A / T)=0$ for all $0 \leqq k<n$.

(3) $\operatorname{Tor}_{k}(X, Y)=0$ for all $0 \leqq k<n$ and for all $Y \in \mathscr{T}_{F}$.

(4) $\operatorname{Ext}^{k}(X, M)=0$ for all $0 \leqq k<n$ and for all $M \in \mathscr{T}_{H}$.

Proof. The proofs of (1) $\Leftrightarrow(2),(1) \Leftrightarrow(3)$, and (1) $\Leftrightarrow(4)$ are dual to the inductive proof of Lemma 1.1. Note that $X \in \mathscr{C}_{1}\left(P_{A}\right)=\mathscr{C}_{H}$ if and only if $X \otimes A / T=0$, if and only if $X \otimes Y=0$ for all $Y \in \mathscr{T}_{F}$, if and only if $\operatorname{Hom}(X, M)=0$ for all $M \in \mathscr{T}_{H}$. Furthermore, for $n>1, X \in \mathscr{C}_{n}\left(P_{A}\right)$ if and only if there is an exact sequence 


$$
0 \rightarrow K \rightarrow U \rightarrow X \rightarrow 0
$$

where $U$ is a direct sum of copies of $P$ and $K \in \mathscr{C}_{n-1}\left(P_{A}\right)$.

If $P_{A}$ is finitely generated projective it is well known that ${ }_{A} P^{*}=$ $\operatorname{Hom}(P, A)$ is finitely generated projective and that $T$ is also the trace ideal of ${ }_{A} P^{*}$. In this case $P \otimes_{A} X \cong \operatorname{Hom}_{A}\left(P^{*}, X\right)$; hence $\mathscr{T}_{F}=$ $\left\{{ }_{A} X \mid \operatorname{Hom}\left(P^{*}, X\right)=0\right\}$ and $\mathscr{C}_{F}=\mathscr{C}_{1}\left({ }_{A} P^{*}\right)$. Thus $T$ is in both $\mathscr{C}_{1}\left(P_{A}\right)$ and $\mathscr{C}_{1}\left({ }_{A} P^{*}\right)$. The following theorem may be of independent interest.

THEOREM 1.2. For $P_{A}$ finitely projective with trace ideal $T, T \in$ $\mathscr{C}_{n}\left(P_{A}\right)$ if and only if $T \in \mathscr{C}_{n}\left({ }_{A} P^{*}\right)$.

Proof. For $k \geqq 1$ we have that $\operatorname{Tor}_{k}(T, A / T) \cong$ $\operatorname{Tor}_{k+1}(A / T, A / T) \cong \operatorname{Tor}_{k}(A / T, T)$. The result follows by Lemma 1.1* and the above remarks.

The right derived functors for a hereditary torsion class $\mathscr{T} \subseteq{ }_{A} \mathcal{M}$ are discussed in [4]. These derived functors are given by $R_{\mathscr{T}}^{0}(X)=t(X)$, $R_{\mathscr{T}}^{1}(X)=t(E(X) / X) / t(E(X))+X / X$, and $R_{\mathscr{T}}^{n}(X)=R_{\mathscr{T}}^{n-1}(E(X) / X)$ for $n \geqq 2$. It is easy to see that $R_{\mathscr{T}}^{1}(X)=0$ for all $X \in \mathscr{T}$. The reader is referred to [16] for further information pertinent to our discussion. The $\operatorname{ring} A$ is said to have $\mathscr{T}-\operatorname{gl} \cdot \operatorname{dim} . A \leqq n$ provided $R_{\mathscr{T}}^{n+1}(X)=0$ for all $X \in{ }_{A} M$.

Let $\mathscr{T} \subseteq{ }_{A} \mathcal{M}$ be a TTF class with $T=c(A)$. The following lemma is an easy consequence of the fact that $t(X) \cong \operatorname{Hom}(A / T, X)$ for any module ${ }_{A} X$.

LEMma 1.3. Let $\mathscr{T} \subseteq{ }_{A} M$ be a TTF class with $T$ the smallest element in $f(\mathscr{T})$. For a module ${ }_{A} X, R_{\mathscr{T}}^{n}(X) \cong \operatorname{Ext}^{n}(A / T, X)$.

COROLlary 1.4. If $\mathscr{T} \subseteq{ }_{A} M$ is a TTF class with $T$ the smallest element in $f(\mathcal{T})$ then $\mathscr{T}-\operatorname{gl} \cdot \operatorname{dim} . A \leqq n$ if and only if h.d. ${ }_{A}(A / T) \leqq n$.

2. Main results. For a hereditary torsion theory $(\mathscr{T}, \mathscr{F}) \subseteq{ }_{A} \mathcal{M}$ cogenerated by the injective module ${ }_{A} V$ the condition that $\mathscr{T}$-gl.dim. $A=$ 0 is examined in [15]. Teply shows [15, Theorem 3.1] that $\mathscr{T}$-gl.dim. $A=0$ if and only if $\mathscr{F}$ is closed under homomorphic images. It is easy to see that $\mathscr{F}$ being closed under homomorphic images is equivalent to $\mathscr{D}_{1}\left({ }_{A} V\right)=$ $\mathscr{D}_{2}\left({ }_{A} V\right)$. In [11] $\mathscr{T}$-gl.dim. $A=0$ is characterized in the special case that $\mathscr{T}$ is TTF. [11, Theorem 1.3] gives several equivalent conditions; e.g., ${ }_{A}(A / T)$ is projective where $T=c(A)$. If $\mathscr{T}=\mathscr{T}_{F}=\left\{{ }_{A} X \mid P \otimes X=0\right\}$ for $P_{A}$ projective [11, Theorem 2.3] equates $\mathscr{T}_{F}$-gl.dim. $A=0$ to conditions on $P$.

The dual situation is also discussed in [11]. Let $\mathscr{T}=\mathscr{T}_{H}=$ $\left\{X_{A} \mid \operatorname{Hom}(P, X)=0\right\}$ for $P_{A}$ projective. It is easily checked that $\mathscr{C}_{1}\left(P_{A}\right)=$ 
$\mathscr{C}_{2}\left(P_{A}\right)$ if and only if $\mathscr{C}_{H}=\mathscr{C}_{1}\left(P_{A}\right)$ is hereditary. Hence [11, Lemma 1.2] gives several conditions equivalent to $\mathscr{C}_{1}\left(P_{A}\right)=\mathscr{C}_{2}\left(P_{A}\right)$; e.g., ${ }_{A}(A / T)$ is flat. [11, Theorem 2.1] relates $\mathscr{C}_{1}\left(P_{A}\right)=\mathscr{C}_{2}\left(P_{A}\right)$ to conditions on $P$.

Throughout the remainder of this paper, unless otherwise noted, $P_{A}$ will be a finitely generated projective $A$-module with trace ideal $T$ and $B=\operatorname{End}\left(P_{A}\right)$. The notation used for the two TTF theories given by $P_{A}$ will be that developed in $\S 1$. The purpose of this section is to discuss the next higher dimensional situation for these TTF theories; i.e., to investigate $\mathscr{D}_{2}\left({ }_{A} V\right)=\mathscr{D}_{3}\left({ }_{A} V\right), \mathscr{T}_{F}$-gl.dim. $A \leqq 1$, and their duals.

The first two conditions of the following theorem are equivalent for an arbitrary hereditary torsion theory (this is essentially [8, Corollary 2.3a]). The first three conditions are equivalent for any TTF-theory.

THEOREM 2.1. Let $\mathscr{T}_{F}=\left\{{ }_{A} X \mid P \otimes X=0\right\}$ be cogenerated by the injective module ${ }_{A} V$. The following are equivalent.

(1) $\mathscr{D}_{2}\left({ }_{A} V\right)=\mathscr{D}_{3}\left({ }_{A} V\right)$.

(2) Given any torsionfree $\mathscr{T}_{F}$-injective module ${ }_{A} X$ and any epimorphism $\theta: X \rightarrow Y$ with $Y \in \mathscr{F}_{F}$, then $Y$ is $\mathscr{T}_{F}$-injective.

(3) $\operatorname{Ext}^{1}\left({ }_{A} T,{ }_{A} N\right)=0$ for all $N \in \mathscr{F}_{F}$.

(4) ${ }_{B} P$ is projective.

Proof. By [6, Theorem 4.5] (2) is equivalent to the localization functor $L_{\mathscr{T}}$ (here $\mathscr{T}=\mathscr{T}_{F}$ ) being exact (see [14] for a discussion of $L_{\mathscr{T}}$ ). Using Lemma 1.1 it is easy to see that $\mathscr{D}_{2}\left({ }_{A} V\right)=\mathscr{D}_{3}\left({ }_{A} V\right)$ if and only if $E(M) / M$ is $\mathscr{T}_{F}$-injective for all torsionfree $\mathscr{T}_{F}$-injective modules $M$. Thus the equivalence of (1) and (2) follows by [8, Corollary 2.3a]. The equivalence of (2) and (4) follows since for $\mathscr{T}=\mathscr{T}_{F}, L_{\mathcal{T}}\left({ }_{A} M\right) \cong$ $\operatorname{Hom}_{B}(P, P \otimes M)$ [3, Proposition 1.6], and $P \otimes_{A} \operatorname{Hom}_{B}(P,-)$ is naturally equivalent to the identity functor on ${ }_{B} \mathcal{M}$. That $(3) \Rightarrow(1)$ follows by Lemma 1.1. We will not prove (2) $\Rightarrow(3)$, but will prove its dual below.

The following provides the dual result. We call a module $X$ $\mathscr{T}$-projective provided $\operatorname{Ext}^{1}(X, M)=0$ for all $M \in \mathscr{T}$.

Theorem 2.1*. For $\mathscr{T}_{H}=\left\{X_{A} \mid \operatorname{Hom}(P, X)=0\right\}$ the following are equivalent.

(1) $\mathscr{C}_{2}\left(P_{A}\right)=\mathscr{C}_{3}\left(P_{A}\right)$.

(2) Given any $\mathscr{T}_{H}$-projective module $X_{A} \in \mathscr{C}_{H}$ and any monomorphism $i: L \rightarrow X$ with $L \in \mathscr{C}_{H}$, then $L$ is $\mathscr{T}_{H}$-projective.

(3) $\operatorname{Tor}_{1}\left(N_{A},{ }_{A} T\right)=0$ for all $N \in \mathscr{C}_{H}$.

(4) ${ }_{B} P$ is flat.

REMARK. The equivalence of (1) through (3) remains valid in the case that $P_{A}$ is not finitely generated. Thus if $A$ is left semihereditary, $\mathscr{C}_{2}\left(U_{A}\right)=\mathscr{C}_{3}\left(U_{A}\right)$ for every projective module $U_{A}$. 
Proof. (1) $\Rightarrow(2)$ By Lemma $1.1^{*} X \in \mathscr{C}_{2}\left(P_{A}\right)$. Let $Y=$ Coker $i$ and consider the exact sequence

$$
\begin{aligned}
\operatorname{Tor}_{2}(Y, A / T) \rightarrow \operatorname{Tor}_{1}(L, A / T) \rightarrow \operatorname{Tor}_{1}(X, A / T) & \rightarrow \operatorname{Tor}_{1}(Y, A / T) \\
& \rightarrow L \otimes A / T .
\end{aligned}
$$

Since both $L \otimes A / T$ and $\operatorname{Tor}_{1}(X, A / T)$ are zero we see that $Y \in$ $\mathscr{C}_{2}\left(P_{A}\right)=\mathscr{C}_{3}\left(P_{A}\right)$; i.e., $\operatorname{Tor}_{2}(Y, A / T)=0$. Thus $\operatorname{Tor}_{1}(L, A / T)=0$ which implies $L$ is $\mathscr{T}_{H}$-projective.

(2) $\Rightarrow$ (3) For $N \in \mathscr{C}_{H}$ there is an epimorphism $\beta: U \rightarrow N$ where $U$ is a direct sum of copies of $P$. Setting $K=\operatorname{Ker} \beta$ we obtain the exact sequence

$$
0 \rightarrow K / K T \rightarrow U / K T \rightarrow N \rightarrow 0
$$

Since $K / K T \otimes T=0$ it is sufficient to show $\operatorname{Tor}_{1}(U / K T, T) \cong$ $\operatorname{Tor}_{2}(U / K T, A / T)=0$. This follows since $U$ is projective, and by assumption, $\operatorname{Tor}_{1}(K T, A / T)=0$.

(3) $\Rightarrow$ (1) Follows by Lemma $1.1^{*}$.

(3) $\Rightarrow$ (4) Let $\alpha: L_{B} \rightarrow M_{B}$ be a monomorphism and let $K_{A}=$ $\operatorname{Ker}\left(\alpha \otimes 1_{P}\right)$. Since ${ }_{B} P \otimes P^{*} \cong{ }_{B} B$ we see that $K \otimes P^{*}=0$; thus $K \otimes T=0$. Setting $I=\operatorname{Im}\left(\alpha \otimes 1_{P}\right)$ we have the commutative diagram

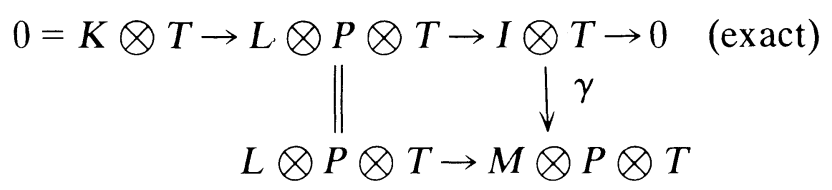

Now $\gamma$ is one-to-one as $(M \otimes P) / I \in \mathscr{C}_{H}$. Hence $\alpha \otimes 1_{P} \otimes 1_{T}$ is one-to-one. The result follows since ${ }_{B} P \otimes T \cong{ }_{B} P$.

(4) $\Rightarrow$ (3) Consider the natural epimorphism $\eta:{ }_{A} P^{*} \otimes P \rightarrow{ }_{A} T$. Since ${ }_{A} K=\operatorname{Ker} \eta \in \mathscr{T}_{F}$ we have, for all $N \in \mathscr{C}_{H}, N \otimes K=N \otimes K / T K \cong$ $N \otimes A / T \otimes K=0$. We conclude $\operatorname{Tor}_{1}(N, T)=0$ since ${ }_{A} P^{*} \otimes P$ is flat [10, Theorem 2.3].

A hereditary torsion theory $(\mathscr{T}, \mathscr{F}) \subseteq{ }_{A} \mathcal{M}$ is said to be perfect provided every left $A_{\mathscr{T}}$-module (where $A_{\mathscr{T}}$ is the left ring of quotients with respect to $\mathscr{T})$ is torsionfree viewed as an $A$-module. Equivalently by $[6, \S 4]\left(\mathscr{T}, \mathscr{F}=\mathscr{D}_{1}\left({ }_{A} V\right)\right)$ is perfect if and only if (i) $\mathscr{D}_{2}\left({ }_{A} V\right)=\mathscr{D}_{3}\left({ }_{A} V\right)$, and (ii) $f(\mathscr{T})$ is $\mathscr{T}$-noetherian (i.e., if $I_{1} \subseteq I_{2} \subseteq \cdots$ is an ascending chain of left ideals whose union is in $f(\mathscr{T})$, then $I_{k} \in f(\mathscr{T})$ for some $\left.k\right)$. In view of Theorem 2.1 it would be interesting to characterize when $f\left(\mathscr{T}_{F}\right)$ is $\mathscr{T}$-noetherian via a condition on ${ }_{B} P$. 

alent.

THEOREM 2.2. For $\mathscr{T}_{F}=\left\{{ }_{A} X \mid P \otimes X=0\right\}$ the following are equiv-

(1) $f\left(\mathscr{T}_{F}\right)$ is $\mathscr{T}$-noetherian.

(2) If ${ }_{B} N_{1} \subseteq{ }_{B} N_{2} \subseteq \cdots$ is an ascending chain of submodules of ${ }_{B} P$ whose union is $P$, then $N_{k}=P$ for some $k$.

(3) $\operatorname{Hom}\left({ }_{B} P,-\right)$ commutes with direct sums.

Proof. By [6, Theorem 4.4] (1) is equivalent to the localization functor $L_{\mathscr{T}_{F}}$ commuting with direct sums. The equivalence of (1) and (3) follows by the same reasons listed in the proof of $(2) \Rightarrow(4)$ of Theorem 2.1 .

(1) $\Rightarrow$ (2) Let $N_{1} \subseteq N_{2} \subseteq \cdots$ be an ascending chain of submodules of ${ }_{B} P$ whose union is $P$. Since $P \cong \operatorname{Hom}\left(P^{*}, A\right)$ we have that ${ }_{A} P^{*} N_{1} \subseteq$ ${ }_{A} P^{*} N_{2} \subseteq \cdots$ is an ascending chain of left ideals of $A$ where $P^{*} N_{i}=$ $\left\{\sum z g \mid z \in P^{*}, g \in N_{i}\right\}$. Now $T=P^{*} P=P^{*}\left(\cup N_{i}\right) \subseteq \cup P^{*} N_{i}$, and thus $\cup P^{*} N_{i} \in f\left(\mathscr{T}_{F}\right)$. So $P^{*} N_{k} \in f\left(\mathscr{T}_{F}\right)$ for some $k$; i.e., $P^{*} N_{k}=T$. Hence $N_{k}=P$ by [13, Theorem 2.2].

(2) $\Rightarrow$ (1) Let $I_{1} \subseteq I_{2} \subseteq \cdots$ be an ascending chain of left ideals of $A$ whose union is in $f\left(\mathscr{T}_{F}\right)$. Then $P I_{1} \subseteq P I_{2} \subseteq \cdots$ is an ascending chain of submodules of ${ }_{B} P$. Since $P=P T \subseteq P\left(\cup I_{i}\right) \subseteq \cup P I_{i}$ we have that $P I_{k}=P$ for some $k$. Thus $I_{k} \in f\left(\mathscr{T}_{F}\right)$ as $P \otimes A / I_{k} \cong P / P I_{k}=0$.

For the TTF class $\mathscr{T}_{F}$ various other torsion theoretic chain conditions can be characterized via conditions on ${ }_{B} P$. For a hereditary torsion class $\mathscr{T}$ with torsion radical $t$ (see [14]), a module $X$ is said to be $t$-noetherian ( $t$-artinian) provided $X$ has ACC (DCC) on closed submodules. $M$ is a closed submodule of $X$ provided $X / M \in \mathscr{F}$. The following theorem is essentially [10, Theorem 3.3].

THEOREM 2.3. Let $(\mathscr{C}, \mathscr{T}, \mathscr{F}) \subseteq{ }_{A} M$ be a TTF theory with $T=c(A)$. For ${ }_{A} X$ there is a one-to-one inclusion preserving correspondence between the submodules of $X$ belonging to $\mathscr{C}$ and the closed submodules of $X$ given by $Y \rightarrow(Y: T)_{X}=\{x \in X \mid T x \subseteq Y\}$. The inverse correspondence is given by $M \rightarrow T M$.

Proof. In view of [10, Theorem 3.3] we must show that $X / M \in \mathscr{F}$ (i.e., $T(x+M)=0$ implies $x \in M$ ) if and only if $(T M: T)_{X} \subseteq M$ (the reverse inclusion is always true). This follows since (using the idempotence of $T) T(x+M)=0$ if and only if $x \in(T M: T)_{X}$.

Corollary 2.4. For $P_{A}$ finitely generated projective

(1) ${ }_{A} A$ is $t_{F}$-artinian ( $t_{F}$-noetherian) if and only if ${ }_{B} P$ is artinian (noetherian). erian).

(2) If ${ }_{A} A$ is $t_{F}$-artinian ( $t_{F}$-noetherian) then ${ }_{B} B$ is artinian (noeth- 
(3) If ${ }_{A} A$ is $t_{F}$-artinian and ${ }_{A} T$ is finitely generated then ${ }_{A} A$ is $t_{F}$-noetherian.

Remarks. (i) Statements (1) and (2) provide a slight generalization of [13, Proposition 2.3].

(ii) For a hereditary torsion class $\mathscr{T} \subseteq{ }_{A} \mathcal{M}$, Manocha [9, Corollary 6.15] has shown that ${ }_{A} A$ being $t$-artinian implies ${ }_{A} A$ is $t$-noetherian provided $\mathscr{T}$ is perfect. In our special case (3), we require only that $f\left(\mathscr{T}_{F}\right)$ contain a cofinal family of finitely generated left ideals.

Proof. The corollary follows easily by [13, Theorem 2.2] and Theorem 2.3. In (2) since ${ }_{A} P^{*}$ is finitely generated we have that ${ }_{A} P^{*}$ is $t_{F}$-artinian $\left(t_{F}\right.$-noetherian) [9]. In (3) ${ }_{B} P$ is finitely generated as ${ }_{A} T$ is. Thus ${ }_{B} P$ is noetherian by (2); and (3) follows by (1).

We now turn our attention to $\mathscr{T}_{F}$-gl.dim. $A \leqq 1$ and its dual. First, we need the following definition.

Definition. We say that a hereditary torsion class $\mathscr{T} \subseteq{ }_{A} \mathcal{M}$ has weak stability if given any injective module ${ }_{A} M$ we have that $M / t(M)$ is $\mathscr{T}$-injective.

Note that if a hereditary torsion class $\mathscr{T}$ is stable then $\mathscr{T}$ has weak stability as every injective module splits. The following theorem provides some characterizations of weak stability.

THEOREM 2.5. For a hereditary torsion class $\mathscr{T} \subseteq{ }_{A} M$ the following are equivalent.

(1) $\mathscr{T}$ has weak stability.

(2) For any $\mathscr{T}$-injective module ${ }_{A} M, M / t(M)$ is $\mathscr{T}$-injective.

(3) $R_{\mathscr{T}}^{2}(X)=0$ for all $X \in \mathscr{T}$.

Furthermore, if $\mathscr{T}$ is a TTF class with $T$ the smallest element in $f(\mathscr{T})$ then each of (1) through (3) are equivalent to any of the following.

(4) ${ }_{A} T$ is $\mathscr{T}$-projective.

(5) For any $\mathscr{T}$-projective module ${ }_{A} U, T U$ is $\mathscr{T}$-projective.

(6) For any projective module ${ }_{A} U, T U$ is $\mathscr{T}$-projective. Finally, any of statements (4) through (6) imply that $A_{\mathscr{T}} \cong \operatorname{End}\left({ }_{A} T\right)$.

REMARKS. (i) If the hereditary torsion class $\mathscr{T}$ has weak stability the localization functor is given by $L_{\mathscr{T}}\left({ }_{A} M\right)=\underline{\lim }_{I \in f(\mathcal{T})} \operatorname{Hom}(I, M)$. This generalizes [14, Proposition 7.7]. The proof is identical to that given in [14] letting $E$ represent the $\mathscr{T}$-injective envelope of $t(M)$ (instead of the injective envelope). One easily checks that $\mathscr{T}$ has weak stability if and only if $\operatorname{Ext}^{2}(A / I, X)=0$ for all $I \in f(\mathscr{T})$ and for every torsion $\mathscr{T}$-injective module $X$.

(ii) $\mathscr{T}$ having weak stability implies that if $N \in \mathscr{F}$ is the 
homomorphic image of a $\mathscr{T}$-injective then $N$ is the homomorphic image of a torsionfree $\mathscr{T}$-injective. This situation also occurs when $A \in \mathscr{F}$. In [3, Theorem 2.1] it is observed for $\mathscr{T}$ a TTF class that $A_{\mathscr{T}} \cong \operatorname{End}(T / t(T))$. Hence if $A \in \mathscr{F}$ then $A_{\mathscr{T}} \cong \operatorname{End}\left({ }_{A} T\right)$.

Proof. (1) $\Rightarrow(3)$ Let $X \in \mathscr{T}$ and let $E_{\mathscr{T}}(X)$ denote its $\mathscr{T}$-injective envelope. By the exact sequence

$$
O=R_{\mathscr{T}}^{1}\left(E_{\mathscr{T}}(X) / X\right) \rightarrow R_{\mathscr{T}}^{2}(X) \rightarrow R_{\mathscr{T}}^{2}\left(E_{\mathscr{T}}(X)\right)
$$

it suffices to show that $R_{\mathscr{T}}^{2}(N)=0$ for any $\mathscr{T}$-injective $N \in \mathscr{T}$. Now $N=t(E(N))$, and so $E(N) / N$ is $\mathscr{T}$-injective by assumption. Hence $R_{\mathscr{T}}^{1}(E(N) / N)=0$. Thus $R_{\mathscr{T}}^{2}(N)=0$ as $R_{\mathscr{T}}^{2}(E(N))=0$.

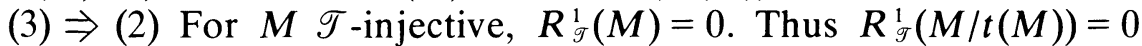
since $R_{\mathscr{T}}^{2}(t(M))=0$ by assumption. This implies that $M / t(M)$ is $\mathscr{T}$ injective as $M / t(M) \in \mathscr{F}$. That $(2) \Rightarrow(1)$ is trivial.

Now let $\mathscr{T}$ be a TTF class. That (5) $\Rightarrow(6)$ is trivial, and $(6) \Rightarrow(4)$ follows since $T A=T$. The equivalence of (3) and (4) follows since for $X \in \mathscr{T}$ we have that $\operatorname{Ext}^{1}(T, X) \cong \operatorname{Ext}^{2}(A / T, X) \cong R_{\mathscr{T}}^{2}(X)$ by Lemma 1.3.

(4) $\Rightarrow(5)$ Let $U$ be $\mathscr{T}$-projective. Since $\operatorname{Hom}(T U, X)=0$ for all $X \in \mathscr{T}$ it follows that $U / T U$ is $\mathscr{T}$-projective. Since $U / T U \in \mathscr{T}$ there is an epimorphism $\alpha: Y \rightarrow U / T U$ where ${ }_{A} Y$ is a direct sum of copies of $A / T$. By (4) we have that $\operatorname{Ext}^{2}(Y, X)=0$ for all $X \in \mathscr{T}$. Thus $\operatorname{Ext}^{2}(U / T U, X)=0$ for all $X \in \mathscr{T}$ since $U / T U$ is a direct summand of $Y$. This implies $T U$ is $\mathscr{T}$-projective as $U$ is $\mathscr{T}$-projective.

Finally, since $\mathscr{T}$ is TTF, $A_{\mathscr{T}}=\operatorname{Hom}(T, A / t(A))$ (see [3]). However, $\operatorname{Hom}(T, A / t(A)) \cong \operatorname{Hom}(T, A) \cong \operatorname{Hom}(T, T)$ where the first isomorphism follows by (4) and the second by the fact that $\operatorname{Hom}(T, A / T)=0$. Therefore, $A_{\mathscr{T}} \cong \operatorname{End}\left({ }_{A} T\right)$.

COROllary 2.6. For $P_{A}$ finitely generated projective the following are equivalent.

(1) $\mathscr{T}_{F}$ has weak stability.

(2) $\mathscr{T}_{H}$ has weak stability.

(3) The natural map $\eta: P^{*} \otimes{ }_{B} P \rightarrow T$ is an (A, A)-isomorphism.

Proof. We prove only (1) $\Leftrightarrow$ (3) as the equivalence of (2) and (3) follows by symmetry.

(1) $\Rightarrow(3){ }_{A} K=\operatorname{Ker} \eta$ is a direct summand of ${ }_{A} P^{*} \otimes P$ as ${ }_{A} T$ is $\mathscr{T}_{F}$-projective. Therefore, $K \in \mathscr{C}_{F} \cap \mathscr{T}_{F}$, which implies $K=0$.

(3) $\Rightarrow$ (1) Since ${ }_{B} B$ is a generator there is an exact sequence

$$
P^{*} \otimes M_{2} \rightarrow P^{*} \otimes M_{1} \rightarrow P^{*} \otimes P \rightarrow 0
$$


where $M_{1}$ and $M_{2}$ are direct sums of copies of ${ }_{B} B$. Thus ${ }_{A} T \cong$ ${ }_{A} P^{*} \otimes P \in \mathscr{C}_{2}\left({ }_{A} P^{*}\right)$. Now $\mathscr{T}_{F}=\left\{{ }_{A} X \mid \operatorname{Hom}\left(P^{*}, X\right)=0\right\}$, and so ${ }_{A} T$ is $\mathscr{T}_{F}$-projective by Lemma $1.1^{*}$. Hence $\mathscr{T}_{F}$ has weak stability by Theorem 2.5.

Our next theorem characterizes $\mathscr{T}$-gl.dim. $A \leqq 1$ for a TTF class $\mathscr{T}$. Weak stability provides the link between $\mathscr{D}_{2}\left({ }_{A} V\right)=\mathscr{D}_{3}\left({ }_{A} V\right)$ and $\mathscr{T}$ $\operatorname{gl} \operatorname{dim} . A \leqq 1$.

THEOREM 2.7. Let $\mathscr{T} \subseteq{ }_{A} \mathcal{M}$ be a TTF class cogenerated by the injective module ${ }_{A} V$ with $T=c(A)$. The following are equivalent.

(1) $\mathscr{T}$-gl.dim. $A \leqq 1$.

(2) $\mathscr{T}$ has weak stability and $\mathscr{D}_{2}\left({ }_{A} V\right)=\mathscr{D}_{3}\left({ }_{A} V\right)$.

(3) ${ }_{A} T$ is projective.

(4) For any projective module ${ }_{A} U, T U$ is projective.

(5) For any $\mathscr{T}$-projective module ${ }_{A} X \in \mathscr{T}$, h.d. $X \leqq 1$.

REMARK. Statement (2) says that any torsionfree homomorphic image of a $\mathscr{T}$-injective module is $\mathscr{T}$-injective. The equivalence of (1) and (2) is true for any hereditary torsion theory and is due to C. Megibben (see [16, Remark ii]).

Proof. The equivalence of (1) and (3) follows by Corollary 1.4. The equivalence of (2) and (3) follows by Theorem 2.1 (3) (with its preceding remark) and Theorem 2.5 (4). That (4) implies (3) is trivial.

(3) $\Rightarrow(5)$ Let $X \in \mathscr{T}$ be $\mathscr{T}$-projective. As in the proof of (4) $\Rightarrow(5)$ of Theorem 2.5, $X$ is a direct summand of a direct sum of copies of $A / T$. Thus h.d. $X \leqq 1$.

(5) $\Rightarrow(4)$ If ${ }_{A} U$ is projective then $U / T U$ is $\mathscr{T}$-projective, and we have that h.d. $U / T U \leqq 1$. Thus $T U$ is projective.

Since ${ }_{A} T$ being finitely generated is equivalent to $f(\mathscr{T})$ containing a cofinal family of finitely generated left ideals we have the following corollary.

Corollary 2.8. For $\mathscr{T} \subseteq{ }_{A} M$ a TTF class with $T=c(A)$ the following are equivalent.

(1) $\mathcal{T}$ is perfect and has weak stability.

(2) ${ }_{\mathrm{A}} T$ is finitely generated projective.

Dualizing, we have the following result.

Theorem 2.7*. For $\mathscr{T}_{H}=\left\{X_{A} \mid \operatorname{Hom}(P, X)=0\right\}$ the following are equivalent.

(1) $\mathscr{T}_{H}$ has weak stability and $\mathscr{C}_{2}\left(P_{A}\right)=\mathscr{C}_{3}\left(P_{A}\right)$.

(2) Given any $\mathscr{T}_{H}$-projective module $X$ and any monomorphism $i: L \rightarrow X$ with $L \in \mathscr{C}_{H}$, then $L$ is $\mathscr{T}_{H}$-projective. 
(3) ${ }_{A} T$ is flat.

(4) For any injective module $M_{A}, M / t_{H}(M)$ is injective.

(5) For any $\mathscr{T}_{H}$-injective module $Y_{A} \in \mathscr{T}_{H}$, injective $\operatorname{dim} . Y \leqq 1$.

Proof. By Corollary 2.6 and Lemma $1.1^{*}, \mathscr{T}_{H}$ has weak stability if and only if $\operatorname{Tor}_{1}(M, T)=0$ for all $M \in \mathscr{T}_{H}$. This fact and Theorems $2.1^{*}$ and 2.5 yield the equivalence of (1) through (3). That (4) is equivalent to (5) is easy.

(3) $\Leftrightarrow(4)$ Both (3) and (4) imply that $\mathscr{T}_{H}$ has weak stability. Thus for $M_{A}$ injective we have $\bar{M}=M / t_{H}(M) \cong \operatorname{Hom}(T, \bar{M}) \cong \operatorname{Hom}(T, M)$ where the first isomorphism follows since $\operatorname{Ext}^{1}(A / T, \bar{M})=0$ and the second since $\operatorname{Ext}^{1}\left(T, t_{H}(M)\right)=0$. The equivalence of (3) and (4) now follows by [12, Lemma 1.3].

In view of Theorems 2.7 and 2.1 we have that $\mathscr{T}_{F}$-gl.dim. $A \leqq 1$ if and only if h.d. ${ }_{B} P=0$ and $\mathscr{T}_{F}$ has weak stability. We conclude this paper with a generalization of this result to higher dimensions, plus give the dual result.

THEOREM 2.9. Let $P_{A}$ be finitely generated projective. Let $n \geqq 1$ and suppose that $R_{\mathscr{T}_{F}}^{k}(X)=0$ for all $X \in \mathscr{T}_{F}, 2 \leqq k \leqq n+1$. Then the following are equivalent.

(1) $\mathscr{T}_{F}$-gl.dim. $A \leqq n+1$.

(2) h.d. ${ }_{B} P \leqq n$ and $R_{\mathscr{F}_{F}}^{n+2}(X)=0$ for all $X \in \mathscr{T}_{F}$.

Proof. By Lemmas 1.3 and $1.1^{*}$ we have that ${ }_{A} T \in \mathscr{C}_{n+1}\left({ }_{A} P^{*}\right)$. Thus there is an exact sequence

$$
X_{n} \stackrel{d_{n}}{\longrightarrow} X_{n-1} \stackrel{d_{n-1}}{\longrightarrow} \cdots \longrightarrow X_{0} \stackrel{d_{0}}{\longrightarrow}{ }_{A} T \longrightarrow 0
$$

where each $X_{i}, 0 \leqq i \leqq n$, is a direct sum of copies of ${ }_{A} P^{*}$. Tensoring with $P_{A}$ yields the exact sequence

$$
P \otimes X_{n} \rightarrow P \otimes X_{n-1} \rightarrow \cdots \rightarrow P \otimes X_{0} \rightarrow P \otimes T \rightarrow 0
$$

where each $P \otimes X_{i}$ is isomorphic to a direct sum of copies of ${ }_{B} B$. Set $K=\operatorname{Ker} d_{n-1}=\operatorname{Im} d_{n}$.

(1) $\Rightarrow$ (2) By Corollary 1.4 h.d. ${ }_{A} T \leqq n$, and thus ${ }_{A} K$ is projective. Hence $K$ is a direct summand of $X_{n}$ which implies that ${ }_{B} P \otimes K=$ $\operatorname{Ker}\left(1 \otimes d_{n-1}\right)$ is projective. Therefore, h.d. ${ }_{B} P \leqq n$ as ${ }_{B} P \otimes T \cong{ }_{B} P$.

(2) $\Rightarrow(1)_{B} P \otimes K$ is projective as h.d. ${ }_{B} P \leqq n$. One easily checks that ${ }_{A} P^{*} \otimes P \otimes K$ is projective. Since $K=\operatorname{Im} d_{n} \in \mathscr{C}_{1}\left({ }_{A} P^{*}\right)$ there is a natural $A$-epimorphism $\eta_{K}: P^{*} \otimes P \otimes K \rightarrow K$ given by $(f \otimes p \otimes k) \eta_{K}=f(p) k$ where $f \in P^{*}, p \in P$, and $k \in K$ (see [10]). Note that $N=\operatorname{Ker} \eta_{K} \in \mathscr{T}_{F}$ 
as $P \otimes N=0$. Therefore, $\operatorname{Ext}^{1}(K, Y)=0$ for all $Y \in \mathscr{F}_{F}$. By a dimension shifting argument we see that $\operatorname{Ext}^{n+1}(T, Y)=0$ for all $Y \in \mathscr{F}_{F}$; i.e., $R_{\mathscr{T}_{F}}^{n+2}(Y)=0$ for all $Y \in \mathscr{F}_{F}$. Since $R_{\mathscr{T}_{F}}^{n+2}(X)=0$ for all $X \in \mathscr{T}_{F}(1)$ follows.

THEOREM 2.9*. Let $P_{A}$ be finitely generated projective. Let $n \geqq 1$ and suppose that $R_{\mathscr{T}_{H}}^{k}(X)=0$ for all $X_{A} \in \mathscr{T}_{H}, 2 \leqq k \leqq n+1$. Then the following are equivalent.

(1) Weak dim. ${ }_{A}(A / T) \leqq n+1$.

(2) Weak dim. ${ }_{B} P \leqq n$ and $R_{\mathscr{T}_{H}}^{n+2}(X)=0$ for all $X_{A} \in \mathscr{T}_{H}$.

Proof. By assumption ${ }_{A} T \in \mathscr{C}_{n+1}\left({ }_{A} P^{*}\right)$. The proof is similar to that of Theorem 2.9 with the obvious modifications; we use the same notation. In $(1) \Rightarrow(2)$, since $K \in \mathscr{C}_{1}\left({ }_{A} P^{*}\right)$ is flat we see that ${ }_{B} P \otimes K$ is flat using [2, Proposition 2.2]. Also, using (1), ${ }_{A} T \in \mathscr{C}_{n+2}\left({ }_{A} P^{*}\right)$; this implies $R_{\mathscr{T}_{H}}^{n+2}(X)=0$ for all $X \in \mathscr{T}_{H}$. In $(2) \Rightarrow(1) R_{\mathscr{T}_{H}}^{n+2}(X)=0$ for all $X \in \mathscr{T}_{H}$ yields ${ }_{A} T \in \mathscr{C}_{n+2}\left({ }_{A} P^{*}\right)$; thus $\operatorname{Tor}_{n+2}(X, A / T)=0$ for all $X \in \mathscr{T}_{H}$. Since weak $\operatorname{dim}{ }_{B} P \leqq n$ we see that ${ }_{A} P^{*} \otimes P \otimes K$ is flat. Therefore $\operatorname{Tor}_{1}(M, K)=0$ for all $M \in \mathscr{C}_{H}$ as $N \in \mathscr{T}_{F}$. By dimension shifting $\operatorname{Tor}_{n+2}(M, A / T) \cong \operatorname{Tor}_{n+1}(M, T)=0$ for all $M \in \mathscr{C}_{H}$.

\section{REFERENCES}

1. F. Anderson, Endomorphism rings of projective modules, Math. Z., 111 (1969), 322-332.

2. S. Chase, Direct products of modules, Trans. Amer. Math. Soc., 97 (1960), 457-473.

3. R. Cunningham, E. Rutter, and D. Turnidge, Rings of quotients of endomorphism rings of projective modules, Pacific J. Math., 41 (1972),.647-667.

4. S. E. Dickson, Direct decompositions of radicals, Proc. Conference on Categorical Algebra at LaJolla in 1965, Springer-Verlag, 1966.

5. J. S. Golan, Localization of Noncommutative Rings, Marcel Dekker, N.Y., 1975.

6. O. Goldman, Rings and modules of quotients, J. Algebra, 13 (1969), 10-47.

7. J. P. Jans, Some aspects of torsion, Pacific J. Math., 15 (1965), 1249-1259.

8. J. Lambek, Localization and completion, J. Pure Appl. Algebra, 2 (1972), 343-370.

9. J. Manocha, Doctoral dissertation, University of Wisconsin, 1973.

10. R. W. Miller, Endomorphism rings of finitely generated projective modules, Pacific J. Math., 47 (1973), 199-220.

11. - TTF classes and quasi-generators, Pacific J. Math., 51 (1974), 499-507.

12. K. Morita, Localizations in categories of modules. I., Math. Z., 114 (1970), 121-144.

13. F. L. Sandomierski, Modules over the endomorphism ring of a finitely generated projective module, Proc. Amer. Math. Soc., 31 (1972), 27-31.

14. B. Stenström, Rings and Modules of Quotients, Lecture Notes in Mathematics \#237, SpringerVerlag, 1971.

15. M. Teply, Homological dimension and splitting torsion theories, Pacific J. Math., 34 (1970), 193-205.

16.

A class of divisible modules, Pacific J. Math., 45 (1973), 653-668.

Received December 17, 1975. Research supported by a Faculty Research Grant from the College of William and Mary. 



\section{PACIFIC JOURNAL OF MATHEMATICS}

\section{EDITORS}

RICHARD ARENS (Managing Editor)

University of California

Los Angeles, California 90024

\author{
R. A. Beaumont \\ University of Washington \\ Seattle, Washington 98105
}

\section{J. DugunduI}

Department of Mathematics University of Southern California Los Angeles, California 90007

D. Gilbarg and J. Milgram

Stanford University

Stanford, California 94305

\section{ASSOCIATE EDITORS}

E. F. BECKENBACH
B. H. NeumanN

F. Wolp
K. YoshidA

\section{SUPPORTING INSTITUTIONS}

UNIVERSITY OF BRITISH COLUMBIA

CALIFORNIA INSTITUTE OF TECHNOLOGY

UNIVERSITY OF CALIFORNIA

MONTANA STATE UNIVERSITY

UNIVERSITY OF NEVADA

NEW MEXICO STATE UNIVERSITY

OREGON STATE UNIVERSITY

UNIVERSITY OF OREGON

OSAKA UNIVERSITY

\author{
UNIVERSITY OF SOUTHERN CALIFORNIA \\ STANFORD UNIVERSITY \\ UNIVERSITY OF HAWAII \\ UNIVERSITY OF TOKYO \\ UNIVERSITY OF UTAH \\ WASHINGTON STATE UNIVERSITY \\ UNIVERSITY OF WASHINGTON \\ AMERICAN MATHEMATICAL SOCIETY
}

The Supporting Institutions listed above contribute to the cost of publication of this Journal, but they are not owners or publishers and have no responsibility for its contents or policies.

Mathematical papers intended for publication in the Pacific Journal of Mathematics should be in typed form or offset-reproduced (not dittoed). double spaced with large margins. Underline Greek letters in red, German in green, and script in blue. The first paragraph or two must be capable of being used separately as a synopsis of the entire paper. Items of the biblography should not be cited there unless absolutely necessary, in which case they must be identified by author and Journal, rather than by item number. Manuscripts, in duplicate, may be sent to any one of the four editors. Please classify according to the scheme of Math. Reviews, Index to Vol. 39. All other communications should be addressed to the managing editor, or Elaine Barth, University of California, Los Angeles, California, 90024.

100 reprints are provided free for each article, only if page charges have been substantially paid. Additional copies may be obtained at cost in multiples of 50 .

The Pacific Journal of Mathematics is issued monthly as of January 1966. Regular subscription rate: $\$ 72.00$ a year (6 Vols., 12 issues). Special rate: $\$ 36.00$ a year to individual members of supporting institutions.

Subscriptions, orders for back numbers, and changes of address should be sent to Pacific Journal of Mathematics, 103 Highland Boulevard, Berkeley, California, 94708.

PUBLISHED BY PACIFIC JOURNAL OF MATHEMATICS, A NON-PROFIT CORPORATION Printed at Jerusalem Academic Press, POB 2390, Jerusalem, Israel.

Copyright (C) 1976 Pacific Journal of Mathematics All Rights Reserved 


\section{Pacific Journal of Mathematics}

\section{Vol. 64, No. $2 \quad$ June, 1976}

Richard Fairbanks Arnold and A. P. Morse, Plus and times............. 297

Edwin Ogilvie Buchman and F. A. Valentine, External visibility ......... 333

R. A. Czerwinski, Bonded quadratic division algebras.............. 341

William Richard Emerson, Averaging strongly subadditive set functions in unimodular amenable groups. II .........................

Lynn Harry Erbe, Existence of oscillatory solutions and asymptotic behavior for a class of third order linear differential equations ............. 369

Kenneth R. Goodearl, Power-cancellation of groups and modules........ 387

J. C. Hankins and Roy Martin Rakestraw, The extremal structure of locally compact convex sets ...................................

Burrell Washington Helton, The solution of a Stieltjes-Volterra integral

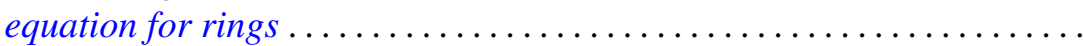

Frank Kwang-Ming Hwang and Shen Lin, Construction of 2-balanced

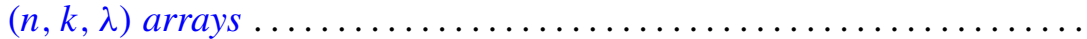

Wei-Eihn Kuan, Some results on normality of a graded ring ... 455

Dieter Landers and Lothar Rogge, Relations between convergence of series and convergence of sequences ......................... 465

Lawrence Louis Larmore and Robert David Rigdon, Enumerating immersions and embeddings of projective spaces ................

Douglas C. McMahon, On the role of an abelian phase group in relativized problems in topological dynamics..................

Robert Wilmer Miller, Finitely generated projective modules and TTF classes...

Yashaswini Deval Mittal, A class of isotropic covariance functions ...

Anthony G. Mucci, Another martingale convergence theorem ...

Joan Kathryn Plastiras, Quasitriangular operator algebras ...

John Robert Quine, Jr., The geometry of $p\left(S^{1}\right) \ldots \ldots \ldots$. 